\title{
An Evaluation Of Single And Dual Long-Acting Bronchodilator Therapy As Effective Interventions In Maintenance Therapy-Naïve Patients With COPD
}

This article was published in the following Dove Press journal:

International Journal of Chronic Obstructive Pulmonary Disease

\author{
Dave Singh' \\ Anthony D D'Urzo² \\ James $\mathrm{F}$ Donohue (D) $^{3}$ \\ Edward M Kerwin (iD ${ }^{4}$ \\ Eduard Molins ${ }^{5}$ \\ Ferran Chuecos ${ }^{5}$ \\ Anna Ribera ${ }^{5}$ \\ Diana Jarreta ${ }^{5}$ \\ 'Medicines Evaluation Unit, University of \\ Manchester, Manchester University NHS \\ Foundation Trust, Manchester, UK; \\ ${ }^{2}$ Department of Family and Community \\ Medicine, University of Toronto, Toronto, \\ ON, Canada; ${ }^{3}$ Division of Pulmonary \\ Diseases and Critical Care Medicine, \\ University of North Carolina Pulmonary \\ Critical Medicine, Chapel Hill, NC, USA; \\ ${ }^{4}$ Clinical Research Institute, Medford, \\ OR, USA; ${ }^{5}$ BioPharmaceuticals R\&D, \\ AstraZeneca, Barcelona, Spain
}

Correspondence: Dave Singh

Medicines Evaluation Unit, University of Manchester, Manchester University NHS

Foundation Trust, Manchester M23 9QZ, UK

Tel +44 I6I 9464073

Fax +44 I6I 9461459

Email DSingh@meu.org.uk
Background: Ideally, treatment recommendations for maintenance therapy-naïve patients with COPD should be based on studies conducted specifically in this population. We have reviewed evidence from previous studies of pharmacological treatments in maintenance therapy-naïve patients with COPD and performed a new post-hoc analysis of dual bronchodilator treatment in this population, aiming to assess the effectiveness of these interventions. Materials and methods: A literature review identified clinical trials that included analyses of patients with COPD who were maintenance therapy-naïve with long-acting $\beta_{2}$-agonists (LABA) or long-acting muscarinic antagonists (LAMA). Additionally, a post-hoc subgroup analysis was conducted for maintenance therapy-naïve patients with COPD in two large phase III, randomized, double-blind, 24-week trials investigating the efficacy of aclidinium bromide/formoterol fumarate $(\mathrm{AB} / \mathrm{FF})$ fixed-dose combination versus monotherapy or placebo (ACLIFORM [NCT01462942] and AUGMENT [NCT01437397]).

Results: Treatment-naïve patients with COPD often represent a population of patients at the earliest stage at which most patients seek treatment. Of nine relevant studies identified, all reported positive findings for efficacy of LABA, LAMA, or LABA/LAMA treatment in maintenance therapy-naïve populations. Improvements were observed in lung function, symptoms, and health status versus monotherapy or placebo. Post-hoc analysis of ACLIFORM and AUGMENT demonstrated that $\mathrm{AB} / \mathrm{FF}$ was effective in improving lung function in patients who had received no prior maintenance therapy. $\mathrm{AB} / \mathrm{FF}$ showed improvements in $1 \mathrm{hr}$ post-dose $\mathrm{FEV}_{1}$, trough $\mathrm{FEV}_{1}$, and patient-reported outcomes versus placebo and monotherapies. Combined with reviews of previous studies in maintenance therapynaïve patients, these findings suggest that earlier intervention with a dual bronchodilator maintenance therapy, such as $\mathrm{AB} / \mathrm{FF}$, may provide significantly greater benefits than LAMA or LABA mono-bronchodilator therapy as a first maintenance treatment for COPD.

Conclusion: These data show that therapeutic intervention is effective in treatment-naïve patients. Intervention with dual bronchodilator therapy as a first maintenance treatment for COPD may provide greater benefits than LAMA or LABA monotherapy.

Keywords: COPD, treatment-naïve, LAMA, LABA

\section{Introduction}

Chronic obstructive pulmonary disease (COPD) is characterized by airflow limitation with persistent respiratory symptoms. ${ }^{1}$ The Global Initiative for Chronic Obstructive Lung Disease (GOLD) report places emphasis on the assessment of 
symptoms and exacerbation risk for newly diagnosed patients. ${ }^{1}$ These parameters enable patients to be categorized using an ABCD assessment, with specific initial pharmacological treatment recommendations defined for each group. ${ }^{1,2}$

The evidence base for these initial treatment recommendations comes from randomized controlled trials (RCTs) that recruited patients already taking maintenance pharmacological treatment and included patients with more advanced disease. Whether the results of these clinical trials apply to newly diagnosed patients with COPD, who are not taking any treatment, is unclear.

RCTs of long-acting bronchodilators often allow patients who are using inhaled corticosteroids (ICS) currently to continue with these drugs. The concurrent use of ICS may influence the treatment effects observed. ${ }^{3}$ Furthermore, the use of maintenance long-acting bronchodilator(s) before the study may result in "step-down" effects being observed after randomization. Recommendations for the initial pharmacological management of newly diagnosed patients with COPD should ideally be based on evidence from patients who were not taking any maintenance treatment before the trial, to avoid step-down effects and/or the confounding effects of ICS use.

This paper aims to evaluate evidence from RCTs and non-interventional observational studies concerning the efficacy of pharmacological treatments in maintenancenaïve patients with COPD. We review studies that enrolled maintenance-naïve patients, and also any post-hoc analyses of this subgroup in RCTs. We also present a new pooled post-hoc subgroup analysis of data from maintenance-naive patients with COPD in the ACLIFORM and AUGMENT studies of aclidinium bromide (AB)/formoterol fumarate (FF) (AB/FF). ${ }^{4,5}$ The results of these two studies have been reported previously, demonstrating the efficacy of $\mathrm{AB} / \mathrm{FF}$ in improving bronchodilation and symptoms versus monotherapy or placebo and also reducing exacerbations versus placebo. ${ }^{4-6}$ Here we analyze the same endpoints in the treatment-naïve patient subgroup, to assess the efficacy of $\mathrm{AB} / \mathrm{FF}$ induced bronchodilation in this sub-population.

\section{Materials And Methods Search Strategy}

To establish the existing evidence base, a literature review was performed to identify clinical studies that included analyses of maintenance therapy-naïve patients with
COPD. Searches in PubMed were conducted for the terms "COPD" and

treatment-naïve/treatment naïve/naïve to maintenance therapy/ naïve to maintenance treatment/maintenance-naïve/maintenance naïve/long-acting $\beta_{2}$-agonist (LABA)-naïve/LABA naïve/long-acting muscarinic antagonist (LAMA)-naïve/ LAMA naïve/prior treatment/prior therapy/inexperienced/ first maintenance.

Further details of the search strategy are shown in Supplementary Figure 1.

Maintenance-naïve was generally defined as naïve to the following maintenance treatments: LABA, LAMA, ICS, or systemic corticosteroids and xanthines. Some studies also excluded short-acting muscarinic antagonists, $\beta_{2}$-agonists plus steroids, bronchodilator combinations, $\beta_{2}$-agonists plus anticholinergic/short-acting muscarinic antagonists and leukotriene antagonists in the months preceding the study. Exclusive use of short-acting $\beta_{2}$-agonists (SABA) was generally permitted prior to study inclusion.

\section{Post-Hoc Analysis Of Patients In ACLIFORM And AUGMENT}

We performed a post-hoc subgroup analysis of maintenance therapy-naïve patients in two large phase III, randomized, double-blind, 24-week trials investigating the efficacy of $\mathrm{AB} /$ FF fixed-dose combination versus either monotherapy or placebo in patients with COPD (ACLIFORM [NCT01462942] and AUGMENT [NCT01437397]). The methods used in ACLIFORM and AUGMENT have been described previously. ${ }^{4,5}$ Briefly, patients with moderate-tosevere stable COPD were randomized to receive $\mathrm{AB} / \mathrm{FF}$ $400 / 12 \mu \mathrm{g}, \mathrm{AB} / \mathrm{FF} 400 / 6 \mu \mathrm{g}, \mathrm{AB} 400 \mu \mathrm{g}, \mathrm{FF} 12 \mu \mathrm{g}$, or placebo twice daily via a multidose dry powder inhaler (Genuair $^{\mathrm{TM}} /$ Pressair $^{\circledR}$ [the registered trademarks of the AstraZeneca group of companies; for use within the USA as Pressair ${ }^{\circledR}$ and Genuair ${ }^{\mathrm{TM}}$ within all other licensed territories]) for 24 weeks. In this pooled post-hoc analysis, treatment-naïve patients were defined as those patients who had not received prior maintenance therapy for COPD: i.e., any LABA, LAMA, ICS, or xanthines; short-acting bronchodilators were permitted. Patients enrolled in the studies were not specifically required to have an established duration of COPD. Prior and concomitant medication data were collected and defined at screening up to approximately 2 weeks prior to randomization, and during the run-in period, resulting in the collection of medication data for at least 4 weeks before the first dose of study medication. Of the two 
$\mathrm{AB} / \mathrm{FF}$ arms, only the currently approved $400 / 12 \mu \mathrm{g}$ dose $^{7}$ is included here. Both the studies included in this post-hoc analysis were conducted in accordance with the Declaration of Helsinki, International Council for Harmonisation/Good Clinical Practice Guidelines, and local regulations. The regulatory authorities approved the protocols for each country and each center had an independent ethics committee.

\section{ACLIFORM And AUGMENT Post-Hoc Statistical Analysis}

Full statistical information for ACLIFORM and AUGMENT has been described previously. ${ }^{4,5}$ The post-hoc analysis presented here included patients who were naïve to maintenance therapy from the pooled intent-to-treat (ITT) population (excluding those allocated to $\mathrm{AB} / \mathrm{FF} 400 / 6 \mu \mathrm{g}$ ). Changes from baseline at Week 24 were analyzed for the following endpoints: pre-dose (trough) and $1 \mathrm{hr}$ morning post-dose forced expiratory volume in 1 second $\left(\mathrm{FEV}_{1} ; \mathrm{mL}\right)$, Transition Dyspnea Index (TDI) focal score, and St George's Respiratory Questionnaire (SGRQ) total score. Changes from baseline in daily symptoms, as measured using the EvaluatingRespiratory Symptoms (E-RS ${ }^{\mathrm{TM}}$ [the E-RS ${ }^{\mathrm{TM}}$ is owned by Evidera. Permission has been granted for this publication. Permission to use this instrument may be obtained from Evidera (exactpro@evidera.com)]; formerly known as EXAcerbations of Chronic pulmonary disease Tool Respiratory Symptoms) total score, ${ }^{8}$ and morning and nighttime symptoms were analyzed over 24 weeks using the Nighttime Symptoms of COPD Instrument, ${ }^{9,10}$ and Early Morning Symptoms of COPD Instrument tools. ${ }^{11}$ All reported data are least squares (LS) mean changes from baseline with 95\% confidence intervals (CI), based on the mixed model for repeated measures: treatment effects and treatment comparisons. LS mean differences between $\mathrm{AB} / \mathrm{FF} 400 / 12 \mu \mathrm{g}$ and treatment groups are also shown $(\Delta)$. For changes from baseline in trough and morning post-dose $\mathrm{FEV}_{1}$, TDI, SGRQ, E-RS, nighttime and early morning outcomes, analyses were adjusted using the main common predictors i.e. screening response $\left(\mathrm{FEV}_{1}\right.$, only), baseline scores and age as covariates, and any of the following criteria as fixed effect factors: treatment group, study, sex, smoking status, visit, prior naïve COPD patients, and interactions of treatment group-by-visit, treatment group-by-prior naïve COPD patients, and treatment group-by-visit-by-prior naïve COPD patients. Minimal clinically important difference (MCID) for each outcome: SGRQ ( $\geq 4$-unit change), ${ }^{12}$ trough $\mathrm{FEV}_{1}$ (change of approximately $100 \mathrm{~mL}){ }^{13}$ TDI focal score (increase of 1 unit), ${ }^{14}$ and
COPD Assessment Test (CAT) score (2-point reduction), ${ }^{15}$ as previously published.

\section{Results \\ Treatment In Maintenance Therapy-Naïve Patients}

The literature search identified nine relevant studies that showed clinical outcomes in a COPD maintenance therapy-naïve patient population or with a COPD maintenance therapy-naïve sub-population (Table 1). Of the nine studies identified, two were dual bronchodilator RCTs, four were long-acting bronchodilator monotherapy RCTs, one was a real-world study of guidelines-based treatment, and two were non-interventional observational studies. All reported positive findings for the efficacy of LABA, LAMA, or LAMA/LABA treatment in maintenance therapy-naïve populations. ${ }^{16-24} \mathrm{Six}$ of the nine studies investigated the lung function effects of bronchodilators in treatment-naïve patients, ${ }^{16,18,20-24}$ while all nine studies investigated symptoms and quality of life (QoL). ${ }^{16-24}$

\section{Long-Acting Bronchodilator Monotherapy Studies}

Four RCTs evaluated lung function, exacerbations, symptoms, and quality of life outcomes for treatment-naïve patients receiving monotherapy with either tiotropium ${ }^{21,22,24}$ or indacaterol. ${ }^{23}$ Tiotropium or indacaterol generally showed significant improvements in a range of lung function measurements versus placebo. ${ }^{21,23,24}$ Compared with placebo, the trough $\mathrm{FEV}_{1}$ improvements for indacaterol $150 \mu \mathrm{g}$ and $300 \mu \mathrm{g}$ were $170 \mathrm{~mL}$ and $180 \mathrm{~mL}$, respectively, $(P<0.001)$ at 6 months (Figure 1A). ${ }^{23}$ In two RCTs of tiotropium versus placebo, the trough $\mathrm{FEV}_{1}$ improvements were $140 \mathrm{~mL}$ at Week $24^{21}$ and $134 \mathrm{~mL}$ after 4 years (both $P<0.001$; Figure 1A). ${ }^{24}$

In treatment-naïve patients at 6 months, indacaterol $150 \mu \mathrm{g}$ and $300 \mu \mathrm{g}$ showed significant improvements $(P<0.001$ and $P<0.05$, respectively) versus placebo in symptoms and health status including SGRQ (treatment difference vs placebo for 150 $\mu \mathrm{g}$ and $300 \mu \mathrm{g}$ of -6.1 and -2.5 units, respectively; $P<0.001$ and $P<0.05$, respectively; Figure $2 \mathrm{~A})$, rescue use $(-0.48$ and -0.59 puffs/day; $P<0.01$ ), and TDI total score (1.27 and 1.04 points; $P<0.001){ }^{23}$ Tiotropium also significantly improved SGRQ versus placebo (treatment difference -4.57 units at 48 months; $95 \%$ CI -7.06 to -2.09 ) (Figure 2A). ${ }^{24}$

There is currently little information available on the exacerbation risk in treatment-naïve patients; however, in one of two analyses of maintenance therapy-naïve patients, tiotropium significantly increased time to first exacerbation 


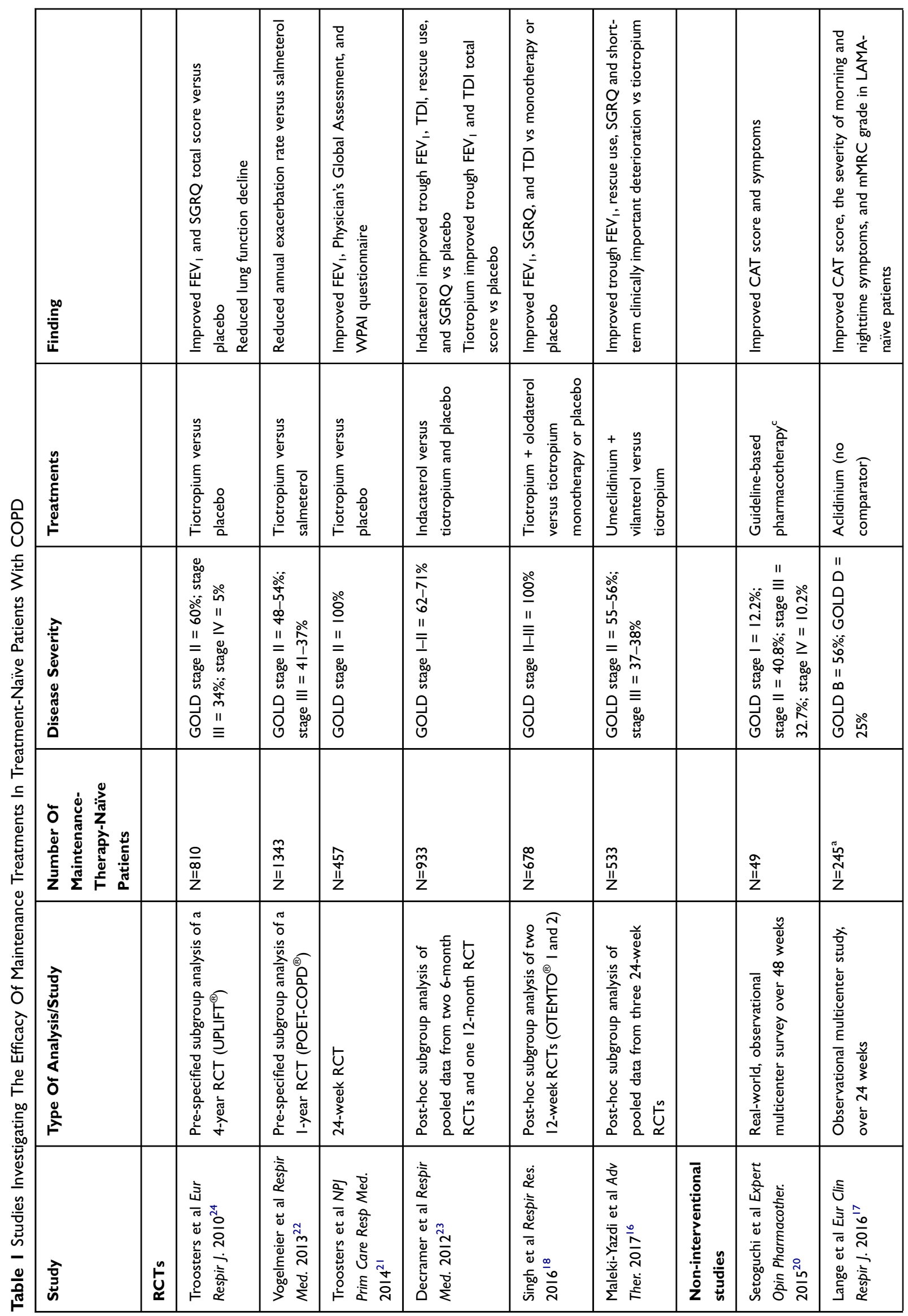




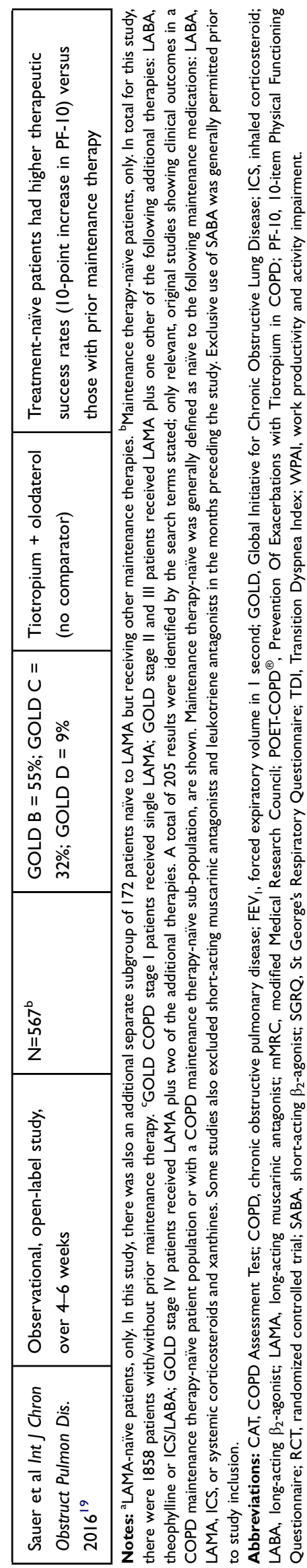

$(P<0.05)$, and reduced annual rates of exacerbations $(P<0.05)$ versus salmeterol, ${ }^{22}$ and the other analyses showed a numerically decreased exacerbation rate by $16 \%(P<0.08$; non-significant $)$ versus placebo. ${ }^{24}$

One study evaluated a decline in lung function over 4 years and showed that tiotropium slowed annual decline compared with placebo (post-bronchodilator $\mathrm{FEV}_{1}$ tiotropium $42 \mathrm{~mL} /$ year vs placebo $53 \mathrm{~mL} /$ year; $P<0.05$ ). ${ }^{24}$ There was also a slowed decline in the activity domain of SGRQ (difference $1.44 \pm 0.40$ units; $P<0.001$; total score difference 4.6 units; $P<0.001$ ) with tiotropium versus placebo over 4 years. ${ }^{24}$

\section{Dual Bronchodilator Studies}

Two studies presented dual bronchodilator outcomes for maintenance therapy-naïve patients as post-hoc analyses. ${ }^{16,18}$ Dual bronchodilation with LAMA/LABA combinations umeclidinium/vilanterol ${ }^{16}$ or tiotropium/olodaterol ${ }^{18}$ provided greater improvements in lung function and health status compared with monotherapy in treatment-naïve patients. Umeclidinium/vilanterol dual bronchodilation showed a significant $146 \mathrm{~mL}$ improvement $(P<0.001)$ from baseline in trough $\mathrm{FEV}_{1}$ versus tiotropium monotherapy at Day 169 (Figure 1A). ${ }^{16}$ Similarly, dual therapy with tiotropium/olodaterol showed improvements in both trough and post-dose $\mathrm{FEV}_{1}$ versus placebo and improvements for post-dose $\mathrm{FEV}_{1}$ versus tiotropium monotherapy, only. For trough $\mathrm{FEV}_{1}$, tiotropium/ olodaterol versus tiotropium monotherapy showed $95 \%$ CIs crossing zero, indicating no significant difference between treatments (Figure 1B). ${ }^{18}$

In terms of QoL, both umeclidinium/vilanterol and tiotropium/olodaterol improved total SGRQ scores from baseline over 12 or 24 weeks ( $\geq 4$-unit decrease), ${ }^{12}$ with both dual therapies resulting in greater improvements compared with tiotropium monotherapy (and also vs placebo for tiotropium/ olodaterol). ${ }^{16,18}$ The effect size of improvement versus tiotropium monotherapy was approximately -2 units for both umeclidinium/vilanterol ${ }^{16}$ and tiotropium/olodaterol (Figure 2B), less than the recognized MCID for SGRQ ( $\geq 4$-unit change). ${ }^{18}$

Symptom measures were reported in one study of tiotropium/olodaterol dual therapy, which showed that TDI focal score was improved versus tiotropium monotherapy with an effect size of approximately 0.5 units at 12 weeks (Figure 2C). ${ }^{18}$ Umeclidinium/vilanterol showed significantly greater proportions ( $47 \%$ ) of patients achieving an increase in clinically meaningful rescue-free periods (one extra rescuefree month per year or two extra rescue-free weeks out of 24) versus tiotropium monotherapy $(37 \%$; odds ratio [OR]: 1.5 [95\% CI: $1.0-2.2])^{16}$ 


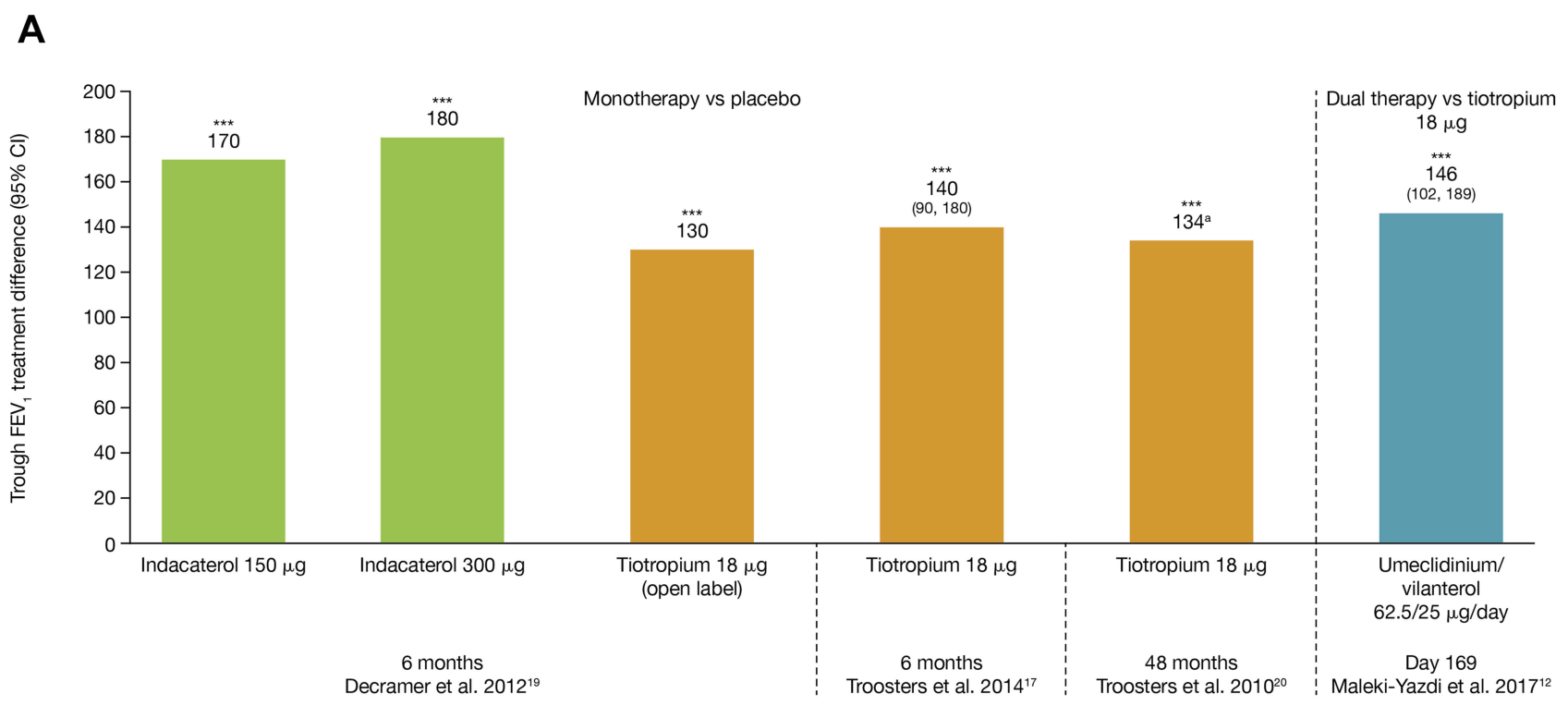

B

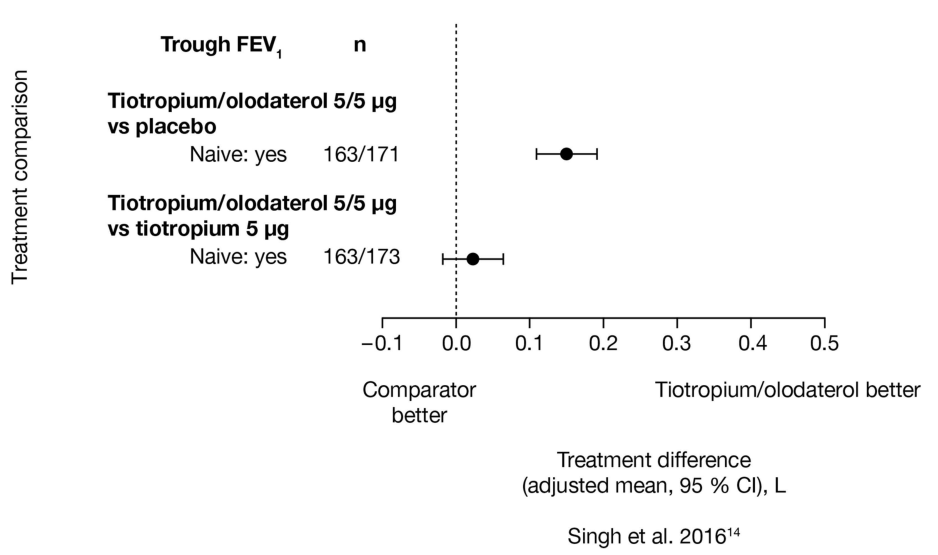

Figure I Treatment differences in trough $\mathrm{FEV}_{\mathrm{I}}$ for $(\mathbf{A})$ monotherapies and $(\mathbf{B})$ dual therapies among the relevant publications identified in the literature search. Notes: Data are least square means differences unless stated otherwise. P-values and $95 \% \mathrm{Cl}$ included where available. ${ }^{\text {a }}$ Mean difference. ${ }^{*} * * * 0.00 \mathrm{I}$ versus placebo. Panel $\mathrm{B}$ is reproduced from the original publication Singh et al $2016,{ }^{18}$ with a simplification of the figure to include only maintenance treatment-naive subgroups. Reproduced from Singh $D$, Gaga M, Schmidt O, et al. Effects of tiotropium + olodaterol versus tiotropium or placebo by COPD disease severity and previous treatment history in the OTEMTO(R) studies. Respir Res. 2016;17(I):73. Creative Commons license and disclaimer available from: http://creativecommons.org/licenses/by/4.0/ and http://creativecommons.org/publicdomain/zero/I.0/. ${ }^{18}$

Abbreviations: $\mathrm{Cl}$, confidence interval; $\mathrm{FEV}_{1}$, forced expiratory volume in I second.

\section{Non-Interventional Studies}

There were three non-interventional, observational studies of bronchodilator therapy in treatment-naïve patients. ${ }^{17,19,20}$ An open-label, multicenter, observational, real-world survey confirmed that initiation of Japanese guideline ${ }^{25}$-directed bronchodilator therapy in Japanese patients with untreated COPD resulted in improved lung function. ${ }^{20}$ GOLD COPD stage I patients received single LAMA, GOLD stage II and III patients received LAMA plus one other of the following additional therapies: LABA, theophylline or ICS/LABA; GOLD stage IV patients received LAMA plus two of the additional therapies.
There were also clinically important differences (2-point reduction $)^{15}$ in mean CAT scores, with improvements from 14.2 at Week 0 to 12.3 at Week $48(P=0.022){ }^{20}$

In a Nordic population of patients with COPD, aclidinium monotherapy in LAMA-naïve patients without concomitant maintenance therapy showed significant improvements from baseline in CAT total score $(-3.8$ mean change $[95 \% \mathrm{CI}-4.6$ to $-3.1] ; P<0.05)$ and exceeded the MCID. There were also improvements from baseline in both morning and nighttime symptoms (both $P<0.01$ ). ${ }^{17}$ For LAMA-naïve patients adding aclidinium to existing maintenance therapy, there were 


\section{A}

Monotherapy vs placebo

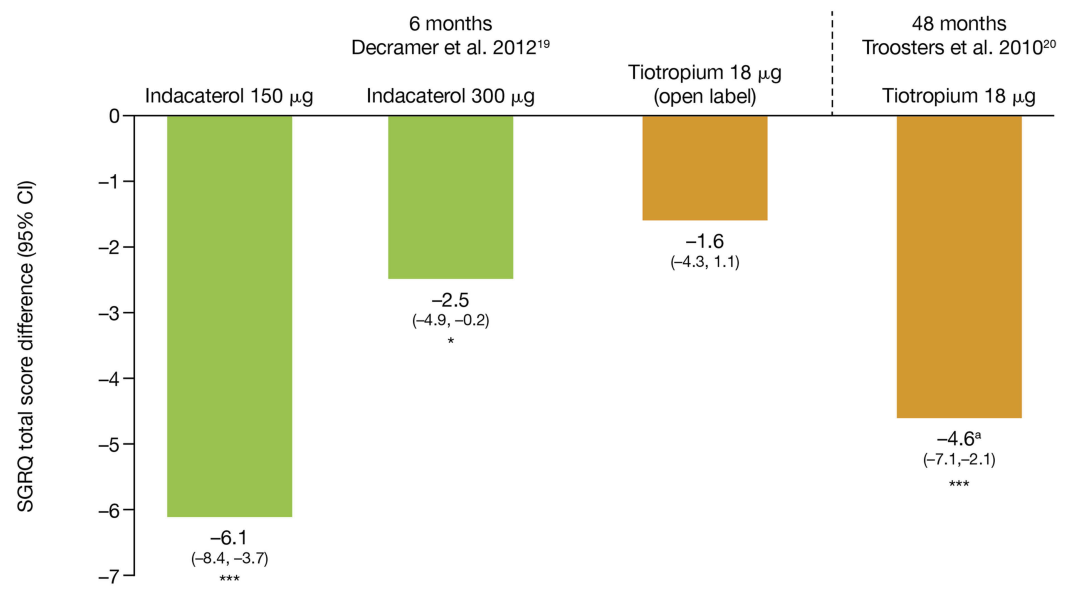

B

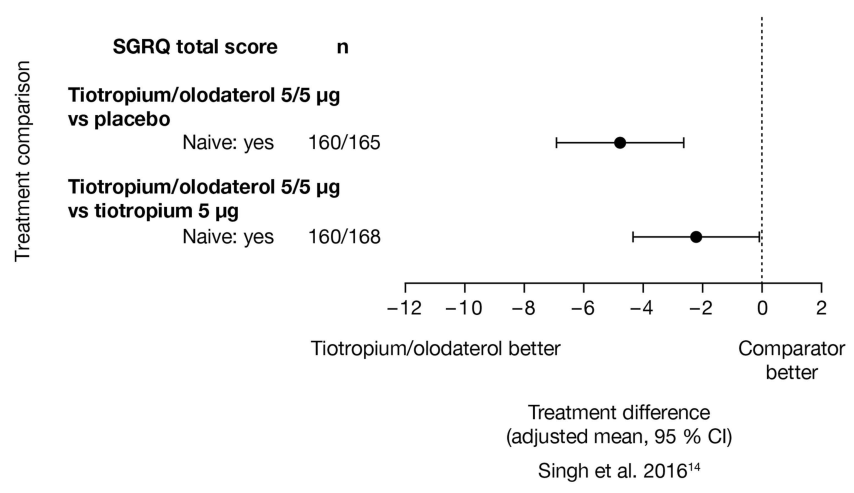

C

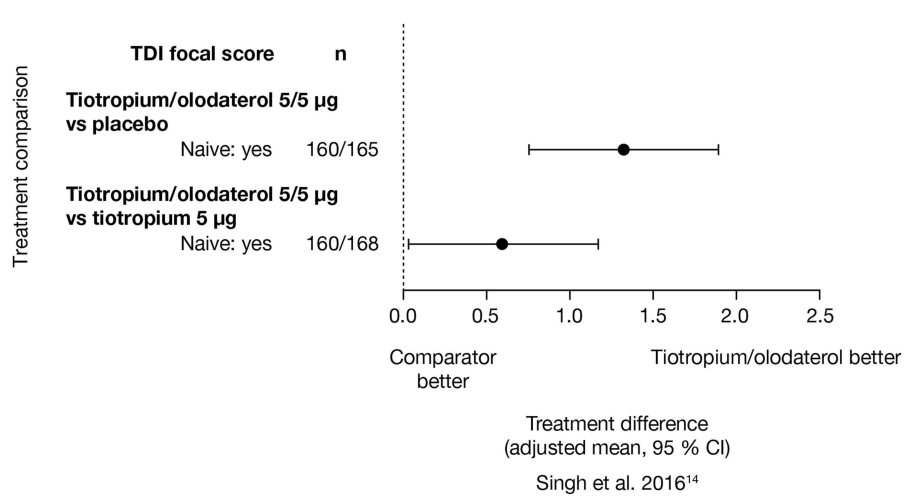

Figure 2 Changes in SGRQ total score for (A) monotherapies and (B) dual therapies; (C) changes in TDI focal score for dual therapies, among the relevant publications identified in the literature search.

Notes: ${ }^{a} S G R Q$ total units. ${ }^{*} P<0.05$; $* * * P<0.001$ versus placebo. Panels $B$ and $C$ are reproduced from the original publication Singh et al 2016 , ${ }^{18}$ with the simplification of the figure to include only maintenance treatment-naïve subgroups. Reproduced from Singh D, Gaga M, Schmidt O, et al. Effects of tiotropium + olodaterol versus tiotropium or placebo by COPD disease severity and previous treatment history in the OTEMTO(R) studies. Respir Res. 2016;17(I):73. Creative Commons license and disclaimer available from: http://creativecommons.org/licenses/by/4.0/ and http://creativecommons.org/publicdomain/zero/I.0/. ${ }^{18}$

Abbreviations: $\mathrm{Cl}$, confidence interval; SGRQ, St George's Respiratory Questionnaire; TDI, Transition Dyspnea Index.

significant improvements from baseline for outcomes including CAT $(-3.3 ; P<0.05)$, modified Medical Research Council
Dyspnea Scale (-0.3), and morning and nighttime symptoms (all $P<0.01) .{ }^{17}$ 
An observational study in patients with COPD receiving dual therapy with tiotropium/olodaterol found that treatment-naïve patients had a higher therapeutic success rate (defined as a 10-point increase in the Physical Functioning Questionnaire between baseline and Weeks 4-6) than those with prior maintenance therapy $(59.1 \%$ vs $44.5 \% ; P<0.0001)$. These differences were driven by a higher response in treatmentnaïve patients who were classified as GOLD B (59.8\%) and $\mathrm{C}(63.0 \%)$; whereas proportions of patients achieving therapeutic success were similar for GOLD D patients, regardless of previous maintenance treatment history. ${ }^{19}$

\section{Efficacy Of AB/FF In Maintenance-}

Therapy-Naïve Patients: A Post-Hoc

Analysis Of Patients In ACLIFORM And AUGMENT

Of 3421 patients in the pooled study population, 3394 were included in the pooled ITT population, and 1056 were naïve to maintenance therapy and included in this analysis (excluding those allocated to $\mathrm{AB} / \mathrm{FF} 400 / 6 \mu \mathrm{g}$ ).

Patient demographics and baseline characteristics of treatment-naïve patients were similar across treatment groups (Table 2). Compared with treatment-exposed patients, treatment-naïve patients were younger, more likely to be

Table 2 Patient Demographics And Baseline Characteristics Of Patients Included In ACLIFORM And AUGMENT (Post-Hoc Analysis; ITT Population)

\begin{tabular}{|c|c|c|c|c|c|c|}
\hline & \multicolumn{4}{|c|}{ Treatment-Naïve } & \multirow{2}{*}{$\begin{array}{l}\text { All } \\
\text { Treatment-Naïve }^{a}\end{array}$} & \multirow{2}{*}{$\begin{array}{l}\text { All } \\
\text { Treatment-Exposed }\end{array}$} \\
\hline & $\begin{array}{l}\text { AB/FF } \\
400 / / 2 \mu g\end{array}$ & $\begin{array}{l}\text { AB } \\
400 \mu g\end{array}$ & $\begin{array}{l}\text { FF } \\
12 \mu g\end{array}$ & Placebo & & \\
\hline & $\mathbf{N}=\mathbf{2 8 2}$ & $\mathbf{N}=\mathbf{2 7 2}$ & $\mathrm{N}=\mathbf{2 7 8}$ & $\mathbf{N}=224$ & $N=1339$ & $\mathbf{N}=\mathbf{2 0 5 5}$ \\
\hline Age, years, mean (SD) & $61.9(8.8)$ & $62.7(8.9)$ & $62.3(8.8)$ & $61.6(8.8)$ & $62.0(8.8)$ & $64.5(8.1)$ \\
\hline Male, n (\%) & $161(57.1)$ & $174(64.0)$ & $168(60.4)$ & $137(6 \mid .2)$ & $818(6 I .1)$ & $1235(60.1)$ \\
\hline White, n (\%) & $256(90.8)$ & $253(93.0)$ & $256(92.1)$ & $211(94.2)$ & $1239(92.5)$ & $1954(95.1)$ \\
\hline $\begin{array}{l}\text { Current smoker, n (\%) } \\
\text { Pack-years, mean (SD) }\end{array}$ & $\begin{array}{l}175(62.1) \\
46.5(23.9)\end{array}$ & $\begin{array}{l}156(57.4) \\
47.0(25.8)\end{array}$ & $\begin{array}{l}162(58.3) \\
47.5(22.2)\end{array}$ & $\begin{array}{l}130(58.0) \\
50.0(25.5)\end{array}$ & $\begin{array}{l}804(60.0) \\
47.7(24.5)\end{array}$ & $\begin{array}{l}872(42.4) \\
45.5(24.2)\end{array}$ \\
\hline $\begin{array}{l}\text { Baseline GOLD } \\
\text { I } \\
\text { II } \\
\text { III } \\
\text { IV }\end{array}$ & $\begin{array}{l}0(0.0) \\
188(66.9) \\
92(32.7) \\
I(0.4)\end{array}$ & $\begin{array}{l}\text { I }(0.4) \\
\text { I } 73(63.8) \\
96(35.4) \\
\text { I }(0.4)\end{array}$ & $\begin{array}{l}0(0.0) \\
189(68.0) \\
86(30.9) \\
3(1.1)\end{array}$ & $\begin{array}{l}0(0.0) \\
127(56.7) \\
95(42.4) \\
2(0.9)\end{array}$ & $\begin{array}{l}2(0.1) \\
867(64.8) \\
460(34.4) \\
8(0.6)\end{array}$ & $\begin{array}{l}3(0.1) \\
1120(54.6) \\
922(45.0) \\
6(0.3)\end{array}$ \\
\hline $\begin{array}{l}\text { Number of exacerbations in previous } \\
12 \text { months, mean (SD) }\end{array}$ & $0.4(0.7)$ & $0.4(0.8)$ & $0.3(0.7)$ & $0.3(0.6)$ & $0.3(0.7)$ & $0.5(0.9)$ \\
\hline Baseline $\mathrm{FEV}_{\mathrm{l}}, \mathrm{L}$, mean $(\mathrm{SD})$ & $\begin{array}{l}1.536 \\
(0.560)\end{array}$ & $\begin{array}{l}I .478 \\
(0.54 I)\end{array}$ & $\begin{array}{l}1.513 \\
(0.513)\end{array}$ & $\begin{array}{l}1.471 \\
(0.6 I I)\end{array}$ & $1.512(0.556)$ & $1.312(0.486)$ \\
\hline Baseline $\mathrm{FEV}_{1}, \%$ predicted, mean (SD) & $\begin{array}{l}\mathrm{N}=282 \\
52.3(14.5)\end{array}$ & $\begin{array}{l}N=27 \mid \\
49.8(14.3)\end{array}$ & $\begin{array}{l}N=278 \\
51.6(14.4)\end{array}$ & $\begin{array}{l}N=223 \\
48.0(14.9)\end{array}$ & $\begin{array}{l}N=1337 \\
50.8(14.5)\end{array}$ & $\begin{array}{l}\mathrm{N}=2055 \\
46.8(13.7)\end{array}$ \\
\hline Reversibility, \%, mean (SD) & $\begin{array}{l}\mathrm{N}=280 \\
12.9(13.3)\end{array}$ & $\begin{array}{l}\mathrm{N}=270 \\
\mathrm{I} 5.8(17.0)\end{array}$ & $\begin{array}{l}\mathrm{N}=278 \\
13.7(\mid 2.9)\end{array}$ & $\begin{array}{l}N=224 \\
16.2(16.0)\end{array}$ & $\begin{array}{l}N=1335 \\
14.6(14.8)\end{array}$ & $\begin{array}{l}\mathrm{N}=2049 \\
\mathrm{I} 5.7(\mid 4.4)\end{array}$ \\
\hline BDI focal score, mean (SD) & $\begin{array}{l}N=273 \\
6.6(2.0)\end{array}$ & $\begin{array}{l}N=264 \\
6.6(2.1)\end{array}$ & $\begin{array}{l}N=27 \mathrm{I} \\
6.6(2.3)\end{array}$ & $\begin{array}{l}N=219 \\
6.6(2.2)\end{array}$ & $\begin{array}{l}N=1299 \\
6.6(2.2)\end{array}$ & $\begin{array}{l}N=1998 \\
6.4(2.1)\end{array}$ \\
\hline Baseline E-RS total score, mean (SD) & $\begin{array}{l}\mathrm{N}=280 \\
\mathrm{II} .8(6.0)\end{array}$ & $\begin{array}{l}\mathrm{N}=270 \\
12.0(6.2)\end{array}$ & $\begin{array}{l}\mathrm{N}=275 \\
\mathrm{II} .9(6.6)\end{array}$ & $\begin{array}{l}\mathrm{N}=219 \\
11.5(6.1)\end{array}$ & $\begin{array}{l}N=1318 \\
11.8(6.3)\end{array}$ & $\begin{array}{l}N=2018 \\
13.1(6.6)\end{array}$ \\
\hline
\end{tabular}

(Continued) 
Table 2 (Continued).

\begin{tabular}{|c|c|c|c|c|c|c|}
\hline & \multicolumn{4}{|c|}{ Treatment-Naïve } & \multirow{2}{*}{$\begin{array}{l}\text { All } \\
\text { Treatment-Naïve }^{a}\end{array}$} & \multirow{2}{*}{$\begin{array}{l}\text { All } \\
\text { Treatment-Exposed }^{\mathrm{a}}\end{array}$} \\
\hline & $\begin{array}{l}\text { AB/FF } \\
400 / 12 \mu g\end{array}$ & $\begin{array}{l}\text { AB } \\
400 \mu g\end{array}$ & $\begin{array}{l}\text { FF } \\
12 \mu g\end{array}$ & Placebo & & \\
\hline & $\mathrm{N}=\mathbf{2 8 2}$ & $\mathbf{N}=\mathbf{2 7 2}$ & $\mathbf{N}=\mathbf{2 7 8}$ & $N=224$ & $N=1339$ & $N=2055$ \\
\hline $\begin{array}{l}\text { Baseline early-morning COPD } \\
\text { symptom severity score, mean (SD) }\end{array}$ & $\begin{array}{l}\mathrm{N}=280 \\
\mathrm{I} .2(0.6)\end{array}$ & $\begin{array}{l}N=270 \\
1.2(0.6)\end{array}$ & $\begin{array}{l}\mathrm{N}=273 \\
\mathrm{I} .2(0.7)\end{array}$ & $\begin{array}{l}N=219 \\
\text { I.I }(0.6)\end{array}$ & $\begin{array}{l}N=1316 \\
1.2(0.6)\end{array}$ & $\begin{array}{l}\mathrm{N}=2023 \\
\mathrm{I} .3(0.7)\end{array}$ \\
\hline $\begin{array}{l}\text { Baseline overall nighttime COPD } \\
\text { symptom severity score, mean (SD) }\end{array}$ & I.I (0.6) & I.I (0.7) & I.I (0.7) & $1.0(0.7)$ & I.I (0.7) & I.I (0.7) \\
\hline Baseline total SGRQ, mean (SD) & $\begin{array}{l}N=276 \\
47.2(17.8)\end{array}$ & $\begin{array}{l}N=264 \\
45.7(16.9)\end{array}$ & $\begin{array}{l}N=275 \\
45.9(18.5)\end{array}$ & $\begin{array}{l}N=221 \\
45.4(17.9)\end{array}$ & $\begin{array}{l}N=1315 \\
46.0(17.9)\end{array}$ & $\begin{array}{l}N=2009 \\
46.2(17.5)\end{array}$ \\
\hline & $N=282$ & $N=272$ & $N=278$ & $N=224$ & $N=1339$ & $N=2055$ \\
\hline Any prior treatment, $\mathrm{n}(\%)$ & $138(48.9)$ & $143(52.6)$ & 137 (49.3) & $110(49.1)$ & $67 \mid(50.1)$ & $2055(100.0)$ \\
\hline SABA & II 5 (40.8) & $109(40.1)$ & III (39.9) & $94(42.0)$ & $549(41.0)$ & $1185(57.7)$ \\
\hline SAMA & $9(3.2)$ & $14(5.1)$ & $9(3.2)$ & $8(3.6)$ & $52(3.9)$ & $148(7.2)$ \\
\hline SABA+SAMA & $24(8.5)$ & $29(10.7)$ & $24(8.6)$ & $19(8.5)$ & $125(9.3)$ & $188(9.1)$ \\
\hline LABA & $0(0.0)$ & $0(0.0)$ & $0(0.0)$ & $0(0.0)$ & $0(0.0)$ & $378(18.4)$ \\
\hline LAMA & $0(0.0)$ & $0(0.0)$ & $0(0.0)$ & $0(0.0)$ & $0(0.0)$ & $909(44.2)$ \\
\hline$L A B A+I C S$ & $0(0.0)$ & $0(0.0)$ & $0(0.0)$ & $0(0.0)$ & $0(0.0)$ & $1059(51.5)$ \\
\hline ICS & $0(0.0)$ & $0(0.0)$ & $0(0.0)$ & $0(0.0)$ & $0(0.0)$ & $478(23.3)$ \\
\hline Xanthines & $0(0.0)$ & $0(0.0)$ & $0(0.0)$ & $0(0.0)$ & $0(0.0)$ & $255(12.4)$ \\
\hline Leukotriene modifiers & $0(0.0)$ & $2(0.7)$ & I (0.4) & I $(0.4)$ & $4(0.3)$ & $4 I(2.0)$ \\
\hline Oxygen & $4(1.4)$ & $2(0.7)$ & $9(3.2)$ & $6(2.7)$ & $26(1.9)$ & $61(3.0)$ \\
\hline Influenza vaccine & $0(0)$ & $4(1.5)$ & $4(1.4)$ & $0(0)$ & $9(0.7)$ & $26(1.3)$ \\
\hline Systemic corticosteroid & $2(0.7)$ & $2(0.7)$ & I $(0.4)$ & $3(1.3)$ & $9(0.7)$ & $24(1.2)$ \\
\hline
\end{tabular}

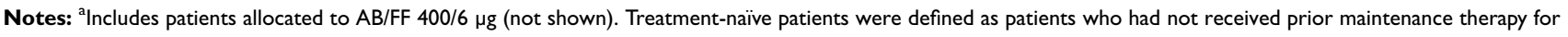
COPD; short-acting bronchodilators were permitted.

Abbreviations: AB, aclidinium bromide; BDI, Baseline Dyspnea Index; COPD, chronic obstructive pulmonary disease; E-RS, Evaluating-Respiratory Symptoms; FEV , forced expiratory volume in I second; FF, formoterol fumarate; ITT, intent-to-treat; SABA, short-acting $\beta_{2}$-agonist; SAMA, short-acting muscarinic antagonist; SD, standard deviation.

current smokers, had higher baseline $\mathrm{FEV}_{1}$ and similar reversibility (Table 2). Treatment-naïve patients had moderately lower rates of prior year exacerbations, lower oxygen, and oral corticosteroid use, and lower E-RS scores at baseline. North American patients were more likely to be treatmentnaïve ( $62.8 \%$ vs $38.9 \%$ non-naïve), whereas European patients were more likely to have received prior maintenance therapy ( $53.1 \%$ vs $31.2 \%$ naïve).

At Week 24, patients receiving $\mathrm{AB} / \mathrm{FF}$ showed significant improvements from baseline in $1 \mathrm{hr}$ post-dose $\mathrm{FEV}_{1}$ (Figure $3 \mathrm{~A}$ ), versus $\mathrm{AB}, \mathrm{FF}$, and placebo (LS mean difference $84 \mathrm{~mL}, 117 \mathrm{~mL}$, and $284 \mathrm{~mL}$, respectively; all $P<0.001$ ). $\mathrm{AB} / \mathrm{FF}$ also showed significantly greater improvement from baseline in trough $\mathrm{FEV}_{1}$ versus $\mathrm{FF}(57 \mathrm{~mL} ; P<0.01)$ and placebo (134 $\mathrm{mL} ; P<0.001$ ) (Figure 3B), although there was no significant difference between $\mathrm{AB} / \mathrm{FF}$ and $\mathrm{AB}(14 \mathrm{~mL}$; $P=0.484$ ). Patients receiving $\mathrm{AB} / \mathrm{FF}$ also had significantly improved changes from baseline in TDI focal scores compared with $\mathrm{AB}(1.17 ; P<0.001)$, FF $(0.92 ; P<0.01)$, and placebo $(1.54 ; P<0.001)$ at Week 24 (Figure 4A).

Change from baseline in E-RS total score was significantly greater with $\mathrm{AB} / \mathrm{FF}$ compared with all other treatments over 24 weeks ( -0.82 and -0.83 vs $\mathrm{AB}$ and $\mathrm{FF}$, both $P<0.05 ;-1.45$ vs placebo, $P<0.001$; Figure $4 \mathrm{~B}$ ). Overall early morning symptom severity was significantly improved from baseline with $\mathrm{AB} / \mathrm{FF}$ compared with $\mathrm{AB}$ $(-0.09 ; P<0.05$; Figure $4 \mathrm{C})$ and placebo $(-0.13 ; P<0.001)$; the improvement versus $\mathrm{FF}$ did not reach statistical significance $(-0.04 ; P=0.317)$. Similarly, overall nighttime symptom severity was significantly improved from baseline with $\mathrm{AB} / \mathrm{FF}$ compared with $\mathrm{AB}(-0.12 ; P<0.01$; Figure 4D), and placebo $(-0.14 ; P<0.001)$; and numerically improved versus FF $(-0.05 ; P=0.20$; non-significant). It is important to note that there was a relatively 

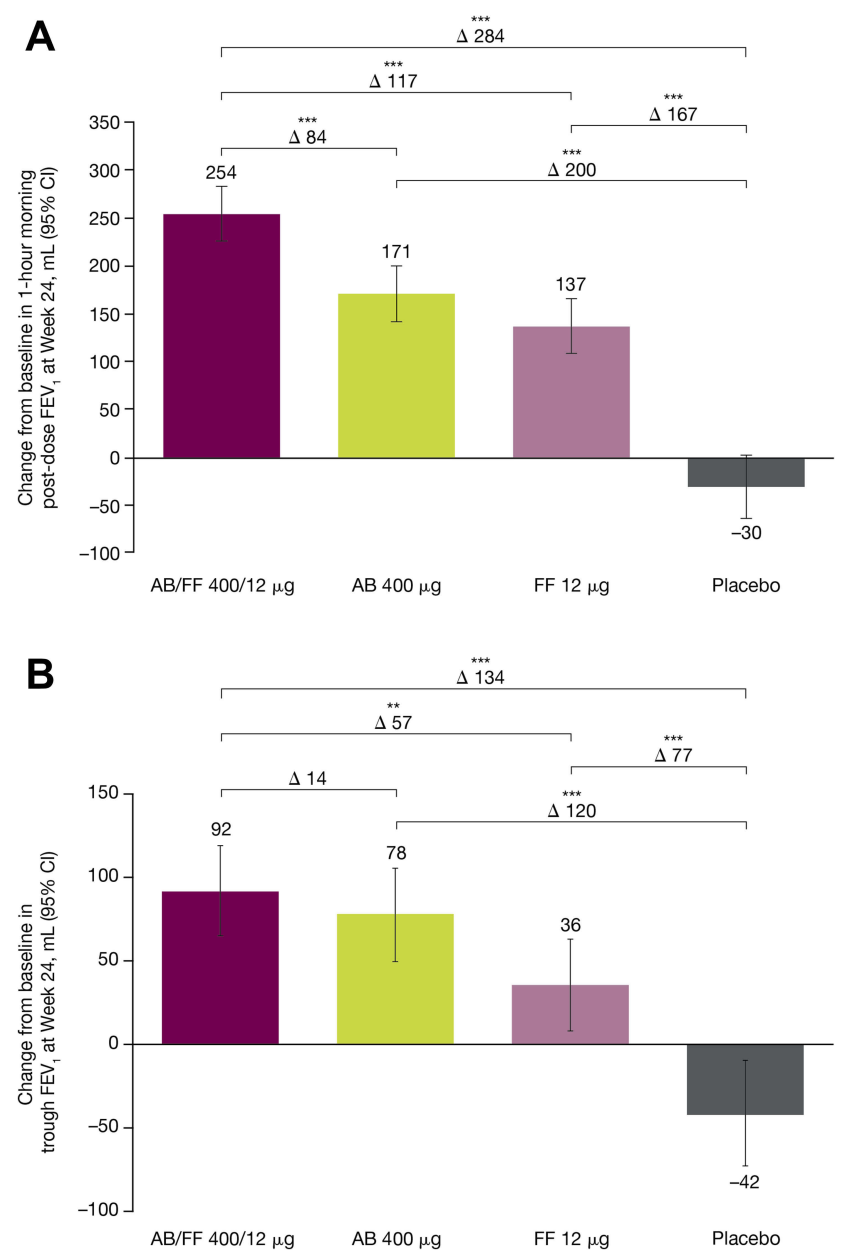

Figure 3 Change from baseline in (A) I hr morning post-dose FEV , and (B) trough FEV , for treatment-naïve patients, at Week 24 of ACLIFORM and AUGMENT (post-hoc analysis; ITT population).

Notes: $* * P<0.01 ; * * * P<0.001$. All data are LS mean changes from baseline. Analyses are based on the mixed model for repeated measures: treatment effects and treatment comparisons. LS mean differences between AB/FF 400/I2 $\mu \mathrm{g}$ and treatment groups are shown $(\Delta)$.

Abbreviations: $A B$, aclidinium bromide; $\mathrm{Cl}$, confidence interval; $\mathrm{FEV} \mathrm{V}_{\mathrm{l}}$, forced expiratory volume in I sec; FF, formoterol fumarate; ITT, intent-to-treat; LS, least squares.

large placebo response observed for TDI focal score, E-RS total score, early morning- and nighttime- symptoms.

Additionally, there were significant improvements from baseline for SGRQ total score at Week 24, for $\mathrm{AB} / \mathrm{FF}$ versus placebo $(-5.3 ; P<0.001), \mathrm{AB}(-3.1 ; \mathrm{P}<0.01)$, and $\mathrm{FF}(-2.3 ; P<0.05)$ (Figure 5). While all active treatment groups exceeded MCID versus baseline, only $\mathrm{AB} / \mathrm{FF}(-5.3)$ exceeded the MCID versus placebo ( $\geq 4$-unit decrease). ${ }^{12}$

\section{Discussion}

A review of the literature revealed several RCTs that included subgroups of treatment-naïve patients. Long-acting bronchodilator monotherapies and dual bronchodilator combinations both showed evidence of significant benefits on lung function, symptoms, and QoL. There was also evidence that LAMA monotherapy appeared to slow the course of $\mathrm{FEV}_{1}$ decline in treatment-naïve patients with COPD, who were mostly GOLD stage II and III. ${ }^{24}$ Furthermore, LAMA/LABA dual bronchodilator therapy provided additional benefits over monotherapy in maintenance therapy-naïve patients. ${ }^{16,18}$ Overall, these data combined with the new post-hoc analysis, support the case for using LAMA/LABA treatments as first-line therapy in COPD.

In general, in terms of lung function, the new post-hoc analysis of the effects of $\mathrm{AB} / \mathrm{FF}$ in maintenance therapynaive patients demonstrated significantly greater benefits than LAMA or LABA mono-bronchodilator therapy. Compared with AB/FF, neither LAMA nor LABA monotherapy treatment was observed to provide similar levels of improvement for airflow (Figure 3) or symptom endpoints (Figure 4). Therefore, these maintenance therapy-naïve patients in ACLIFORM and AUGMENT appeared to benefit most from initial dual bronchodilation with $\mathrm{AB} / \mathrm{FF}$, which provided a combination of anticholinergic and sympathomimetic mechanisms to improve their COPD.

Furthermore, at Week 24, the improvement with $\mathrm{AB} / \mathrm{FF}$ versus placebo in trough $\mathrm{FEV}_{1}(134 \mathrm{~mL})$ exceeded the MCID $(100 \mathrm{~mL}) .{ }^{13} \mathrm{AB} / \mathrm{FF}$ also improved patient-reported outcomes in these maintenance therapy-naïve patients to a greater extent than either of the monotherapies. At Week 24, patients receiving $\mathrm{AB} / \mathrm{FF}$ had significantly improved TDI focal scores compared with monotherapies and placebo, with the improvement versus monotherapies approximately equal to the MCID (1 unit); the treatment differences versus $\mathrm{AB}$ and $\mathrm{FF}$ were 1.17 and 0.92 , respectively. ${ }^{14,26}$ Other symptom and QoL analyses showed a similar pattern of results, supporting a greater effect of $\mathrm{AB} / \mathrm{FF}$ versus monotherapies. These new data support the case that LAMA/ LABA combinations can have a greater effect than longacting bronchodilator monotherapies when used as initial treatment. GOLD recommends that initial treatment with LAMA/LABA should be reserved for individuals with a higher symptom burden. ${ }^{2}$ However, there is a lack of evidence identifying the clinical characteristics that could help identify which patients are most likely to benefit from initial treatment with dual bronchodilators; this topic needs further investigation in terms of prospective studies.

A retrospective database analysis in the UK investigated real-life prescribing of first maintenance therapy in COPD from 2009-2012, and observed that the most frequent first 

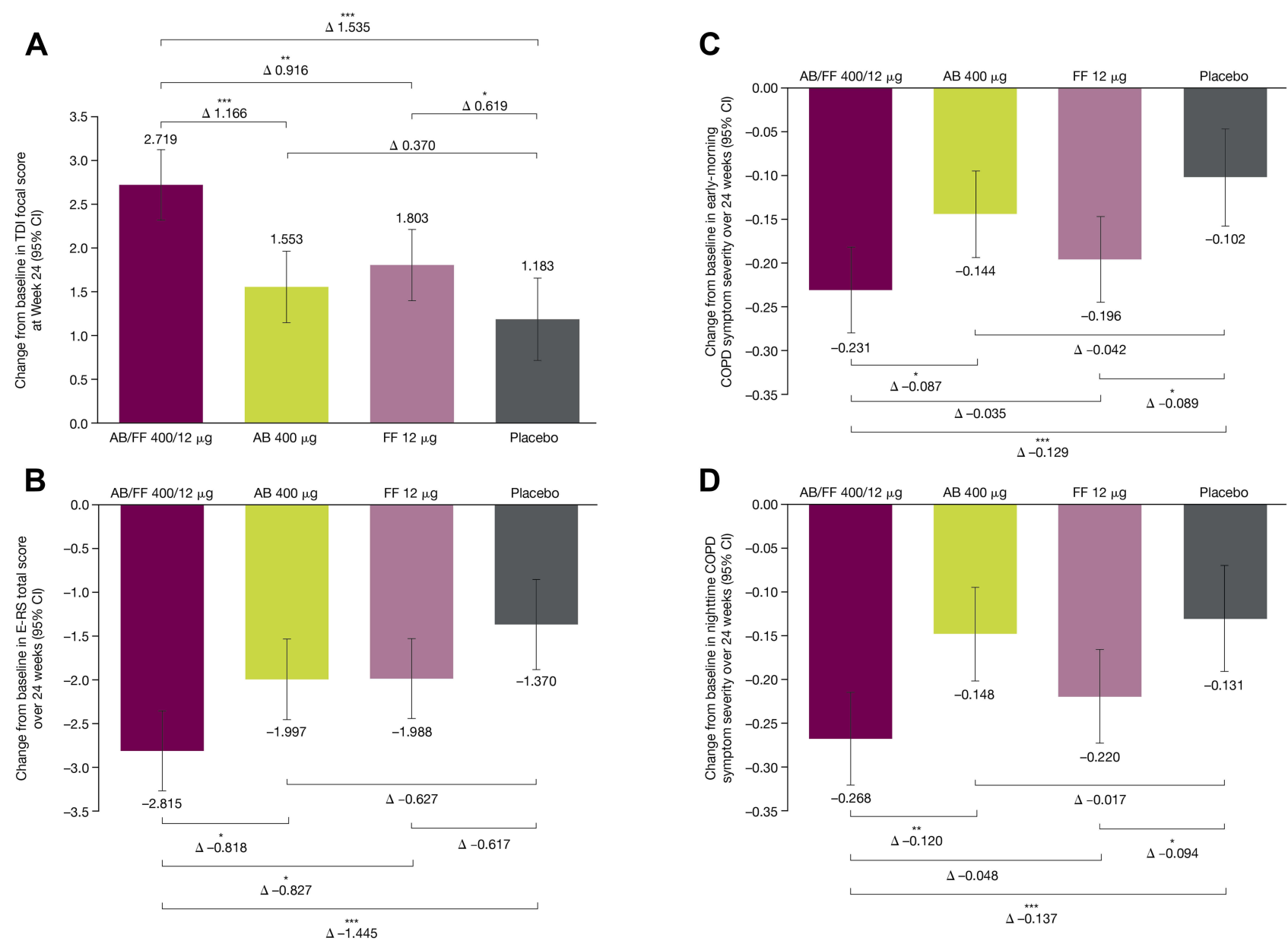

Figure 4 Patient-reported outcomes changes from baseline for treatment-naïve patients (A) TDI focal score at Week 24, (B) E-RS total score, (C) early morning COPD symptom severity and (D) nighttime COPD symptom severity over 24 weeks of ACLIFORM and AUGMENT (post-hoc analysis; ITT population).

Notes: $* P<0.05 ; * * P<0.01 ; * * * P<0.001$. All data are LS mean changes from baseline. Analyses are based on the mixed model for repeated measures: treatment effects and treatment comparisons. LS mean differences between AB/FF 400/12 $\mu \mathrm{g}$ and treatment groups are shown $(\Delta)$.

Abbreviations: $A B$, aclidinium bromide; Cl, confidence interval; COPD, chronic obstructive pulmonary disease; E-RS, Evaluating-Respiratory Symptoms; FF, formoterol fumarate; LS, least squares; TDI, Transition Dyspnea Index.

prescription was for LAMA (40.2\%), followed by ICS +LABA (29.1\%), and ICS monotherapy (15.5\%); only $0.4 \%$ of patients had a first prescription of LAMA + LABA. $^{27}$ However, this was before the introduction of LAMA/LABA combinations in a single inhaler. Additionally, $47.9 \%$ of patients initially assessed as GOLD group A and $49.0 \%$ of patients assessed as GOLD group B received an initial prescription of an ICS alone, or in combination with a bronchodilator. ${ }^{27}$ This illustrates the frequent use of ICS/LABA as an initial COPD prescription. A recent observational study of COPD exacerbation risk with LAMA versus $\mathrm{LABA} / \mathrm{ICS}$ found that $\mathrm{LABA} / \mathrm{ICS}$ was more effective than LAMA in patients with high blood eosinophil concentrations $(>4 \%)$ or counts ( $>300$ cells per $\mu \mathrm{L})$ and also potentially for patients with more frequent exacerbations. ${ }^{28}$ LAMA and LABA/ICS showed similar effectiveness among patients with eosinophil concentrations $<4 \%$, but the elevated pneumonia risk associated with the ICS component of LABA/ICS indicates that initiation with LAMA is more appropriate for patients with low eosinophil counts; although, it should be noted this analysis did not stratify patients by COPD severity or eosinophil count. ${ }^{28}$

One study of dual tiotropium and olodaterol therapy showed that effect sizes for trough $\mathrm{FEV}_{1}$ and $\mathrm{FEV}_{1}$ area under the curve from 0-3 hrs were modestly higher for maintenance therapy-treated versus maintenance therapynaïve patients, but the magnitude of effect of SGRQ total score and TDI focal improvements were similar irrespective of treatment history. ${ }^{18}$ The practical argument in favor of earlier intervention with dual bronchodilator therapy, as opposed to initiating treatment with monotherapy and later escalating to dual therapy, is that it enables an earlier 


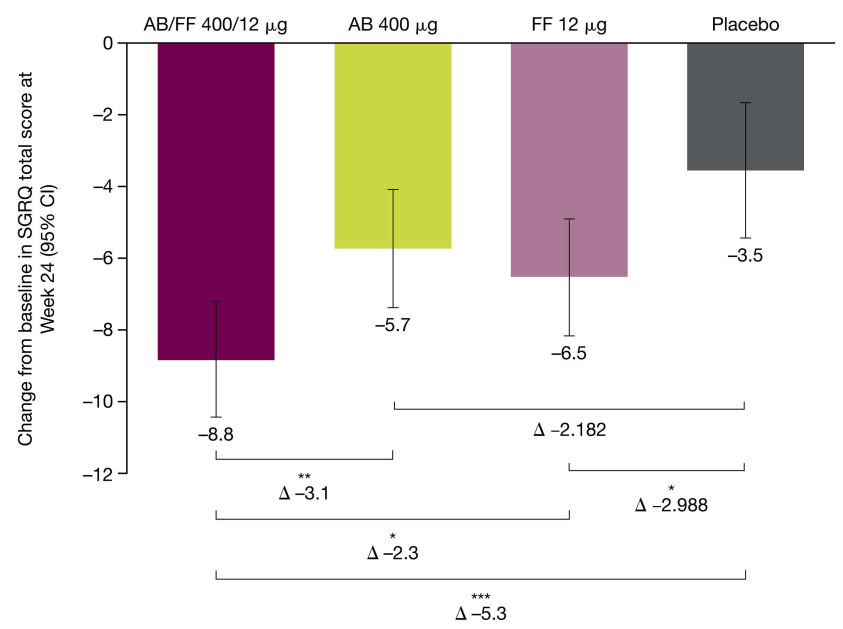

Figure 5 Changes from baseline for SGRQ total score for treatment-naïve patients, at Week 24 of ACLIFORM and AUGMENT (post-hoc analysis; ITT population). Notes: $* P<0.05$; $* * P<0.01$; $* * * P<0.00$ I. All data are LS mean changes from baseline. LS mean differences between AB/FF 400/12 $\mu \mathrm{g}$ and treatment groups are shown $(\Delta)$. The analysis was based on a mixed model for repeated measures: treatment effects and treatment comparisons.

Abbreviations: $\mathrm{AB}$, aclidinium bromide; $\mathrm{Cl}$, confidence interval; $\mathrm{FF}$, formoterol fumarate; ITT, intent to treat; SGRQ, St George's Respiratory Questionnaire.

reduction in the overall disease burden, and could potentially reduce the risk or rate of disease progression in some patients. $^{4,16,29}$ Furthermore, early intervention in COPD may benefit patients as they can maintain levels of activity. ${ }^{30}$

A limitation of the existing evidence for efficacy of maintenance therapy in treatment-naïve patients is that patients included in the studies identified here had a wide range of disease severity from mild to very severe, suggesting that although these patients are receiving treatment for the first time, they may have been living with undiagnosed COPD for a considerable period. This precludes definitive comparison between studies. Additionally, the possibility of a publication bias exists, wherein studies showing significant results may be more likely to be published and therefore identified in our search. As with most of the other studies, our analysis was post-hoc and used a specific definition of maintenance therapy-naïve ( $\geq 4$ weeks of no use of maintenance COPD medications). As such, at least some patients may have used maintenance treatments in the past, which although not received at the time of the current studies, meant there was a possibility of carry-over effects from prior treatments being observed in these studies. Additionally, our maintenance therapy-naïve patients were younger, with higher $\mathrm{FEV}_{1}$, and higher levels of current smoking than maintenance therapytreated patients. These baseline differences might suggest the maintenance therapy-naïve group may be at an earlier, less severe stage of COPD than those already receiving maintenance therapy. There is a need for more prospective studies in patients diagnosed earlier and with milder COPD to evaluate the benefits of introducing maintenance therapy, including dual bronchodilator therapy, at an earlier stage in the development of COPD.

\section{Conclusions}

The findings presented here from retrospective analysis in treatment-naïve patients with COPD, demonstrate the effectiveness of various therapeutic interventions in this population. Of importance, intervention with dual bronchodilator therapy, such as $\mathrm{AB} / \mathrm{FF}$, as a first COPD maintenance treatment may provide greater benefits than LAMA or LABA monotherapy.

\section{Abbreviations}

$\mathrm{AB} / \mathrm{FF}$, aclidinium bromide/formoterol fumarate; CAT, COPD Assessment Test; CI, confidence interval; COPD, chronic obstructive pulmonary disease; E-RS, Evaluating Respiratory Symptoms; $\mathrm{FEV}_{1}$, forced expiratory volume in 1 second; GOLD, Global Initiative for Chronic Obstructive Lung Disease; ICS, inhaled corticosteroid; ITT, intent-to-treat; LABA, long-acting $\beta_{2}$-agonist; LAMA, long-acting muscarinic antagonist; LS, least squares; MCID, minimal clinically important difference; mMRC, modified Medical Research Council; OR, odds ratio; PF-10, 10-item Physical Functioning Questionnaire; POET-COPD ${ }^{\circledR}$, Prevention Of Exacerbations with Tiotropium in COPD; QoL, quality of life; RCT, randomized controlled trial; SABA, short-acting $\beta_{2}$-agonist; SGRQ, St George's Respiratory Questionnaire; TDI, Transition Dyspnea Index; WPAI, work productivity and activity impairment.

\section{Ethics Approval And Informed Consent}

Both the studies included in this post-hoc analysis were conducted in accordance with the Declaration of Helsinki, International Council for Harmonisation/Good Clinical Practice Guidelines, and local regulations. The regulatory authorities approved the protocols for each country and each center had an independent ethics committee.

\section{Data Availability}

Data underlying the findings described in this manuscript may be obtained in accordance with AstraZeneca's data sharing policy described at: https://astrazenecagrouptrials. pharmacm.com/ST/Submission/Disclosure. 


\section{Acknowledgments}

Medical writing support, under the direction of the authors, was provided by Nina Divorty, $\mathrm{PhD}$, and Rebecca J. Douglas, PhD, of CMC Connect, a division of McCann Health Medical Communications Ltd., Glasgow, and Macclesfield, UK and was funded by AstraZeneca, Cambridge, UK in accordance with Good Publication Practice (GPP3) guidelines (Ann Intern Med 2015;163:461-464). This work was funded by AstraZeneca, Cambridge, UK, with the original ACLIFORM and AUGMENT studies funded by Almirall S.A. and Forest Laboratories LLC. The sponsors did not place any restriction on authors about the statements made in the final article.

\section{Author Contributions}

All authors contributed to data analysis, drafting or revising the article, gave final approval of the version to be published, and agree to be accountable for all aspects of the work.

\section{Disclosure}

DS is supported by the NIHR Manchester Biomedical Research Centre; and has received sponsorship to attend international meetings, honoraria for lecturing or attending advisory boards and research grants from various pharmaceutical companies including Apellis, AstraZeneca, Boehringer Ingelheim, Chiesi Farmaceutici S.p.A, Cipla, Genentech, GlaxoSmithKline, Glenmark, Johnson \& Johnson, Mundipharma, Novartis, Peptinnovate Ltd., Pfizer Inc, Pulmatrix, Skyepharma, Teva, Theravance Biopharma, Menarini, and Verona Pharma. ADD has received research, consulting, and lecturing fees from Almirall, Altana, AstraZeneca, Boehringer Ingelheim (Canada) Ltd, Forest Laboratories, GlaxoSmithKline, KOS Pharmaceuticals, Merck Canada, Methapharm, Novartis Canada/USA, ONO Pharmaceutical Co., Pfizer Canada, Schering-Plough, Sepracor, and SkyePharma.

JFD has received consulting fees from AstraZeneca, Boehringer Ingelheim, Chiesi, Circassia, GSK, Mylan, Theravance, and Sunovion. He is a member of the Data Monitoring Committee for AstraZeneca.

EMK has participated in consulting, advisory boards, speaker panels, or received travel reimbursement from Amphastar Pharmaceuticals, AstraZeneca, Boehringer Ingelheim, Cipla, Chesi, Forest Laboratories LLC, GSK, Mylan, Novartis, Oriel, Pearl, Sunovion, Teva
Pharmaceutical Industries Ltd., and Theravance Biopharma. He has conducted multicenter clinical research trials for $\sim 40$ pharmaceutical companies. EM, FC, and DJ are employees of AstraZeneca and former employees of Almirall S.A., Barcelona, Spain. AR is a former employee of Almirall S.A., Barcelona, Spain and was an employee of AstraZeneca at the time the study was conducted. The authors report no other conflicts of interest in this work.

\section{References}

1. Vogelmeier CF, Criner GJ, Martinez FJ, et al. Global strategy for the diagnosis, management, and prevention of chronic obstructive lung disease 2017 report: GOLD executive summary. Eur Respir J. 2017;49(3):1700214. doi:10.1183/13993003.00214-2017

2. Singh D, Agusti A, Anzueto A, et al. Global strategy for the diagnosis, management, and prevention of chronic obstructive lung disease: the GOLD Science Committee report 2019. Eur Respir J. 2019;53:1900164. doi:10.1183/13993003.00164-2019

3. Magnussen H, Disse B, Rodriguez-Roisin R, et al. Withdrawal of inhaled glucocorticoids and exacerbations of COPD. $N$ Engl J Med. 2014;371(14):1285-1294. doi:10.1056/NEJMoa1407154

4. Singh D, Jones PW, Bateman ED, et al. Efficacy and safety of aclidinium bromide/formoterol fumarate fixed-dose combinations compared with individual components and placebo in patients with COPD (ACLIFORM-COPD): a multicentre, randomised study. $B M C$ Pulm Med. 2014;14(1):178. doi:10.1186/1471-2466-14-178

5. D'Urzo AD, Rennard SI, Kerwin EM, Mergel V, Leselbaum AR, Caracta CF. Efficacy and safety of fixed-dose combinations of aclidinium bromide/formoterol fumarate: the 24-week, randomized, placebo-controlled AUGMENT COPD study. Respir Res. 2014;15 (1):123. doi:10.1186/s12931-014-0123-0

6. Bateman ED, Chapman KR, Singh D, et al. Aclidinium bromide and formoterol fumarate as a fixed-dose combination in COPD: pooled analysis of symptoms and exacerbations from two six-month, multicentre, randomised studies (ACLIFORM and AUGMENT). Respir Res. 2015;16:92. doi:10.1186/s12931-015-0250-2

7. AstraZeneca PLC. Duaklir ${ }^{\circledR}$ Genuair ${ }^{\mathrm{TM}}$ Summary of Product Characteristics. 2018. Available from: http://www.medicines.org.uk/ emc/medicine/29652. Accessed June 22, 2018.

8. Leidy NK, Murray LT, Monz BU, et al. Measuring respiratory symptoms of COPD: performance of the EXACT-Respiratory Symptoms Tool (E-RS) in three clinical trials. Respir Res. 2014;15(1):124. doi:10.1186/s12931-014-0124-z

9. Hareendran A, Palsgrove AC, Mocarski M, et al. The development of a patient-reported outcome measure for assessing nighttime symptoms of chronic obstructive pulmonary disease. Health Qual Life Outcomes. 2013;11:104. doi:10.1186/1477-7525-11-104

10. Mocarski M, Zaiser E, Trundell D, Make BJ, Hareendran A. Evaluation of the psychometric properties of the Nighttime Symptoms of COPD Instrument. Int J Chron Obstruct Pulmon Dis. 2015;10:475-487. doi:10.2147/COPD.S75776

11. Mocarski M, Hareendran A, Jen MH, Zaiser E, Make B. Evaluation of the psychometric properties of the Early Morning Symptoms of COPD Instrument (EMSCI). Value Health. 2014;17:A179.

12. Jones PW. St. George's Respiratory Questionnaire: MCID. COPD. 2005;2(1):75-79. doi:10.1081/COPD-200050513

13. Donohue JF. Minimal clinically important differences in COPD lung function. COPD. 2005;2(1):111-124. doi:10.1081/COPD-200053377

14. Mahler DA, Witek TJ Jr. The MCID of the transition dyspnea index is a total score of one unit. COPD. 2005;2(1):99-103. doi:10.1081/ COPD-200050666 
15. Kon SSC, Canavan JL, Jones SE, et al. Minimum clinically important difference for the COPD Assessment Test: a prospective analysis. Lancet Respir Med. 2014;2(3):195-203. doi:10.1016/S2213-2600(14)70001-3

16. Maleki-Yazdi MR, Singh D, Anzueto A, Tombs L, Fahy WA, Naya I. Assessing short-term deterioration in maintenance-naive patients with COPD receiving umeclidinium/vilanterol and tiotropium: a pooled analysis of three randomized trials. Adv Ther. 2017;33(12):21882199. doi:10.1007/s12325-016-0430-6

17. Lange P, Godtfredsen NS, Olejnicka B, et al. Symptoms and quality of life in patients with chronic obstructive pulmonary disease treated with aclidinium in a real-life setting. Eur Clin Respir J. 2016;3:31232. doi:10.3402/ecrj.v3.31232

18. Singh D, Gaga M, Schmidt O, et al. Effects of tiotropium + olodaterol versus tiotropium or placebo by COPD disease severity and previous treatment history in the OTEMTO(R) studies. Respir Res. 2016;17(1):73. doi:10.1186/s12931-016-0387-7

19. Sauer R, Hansel M, Buhl R, Rubin RA, Frey M, Glaab T. Impact of tiotropium + olodaterol on physical functioning in COPD: results of an open-label observational study. Int J Chron Obstruct Pulmon Dis. 2016;11:891-898. doi:10.2147/COPD.S103023

20. Setoguchi Y, Izumi S, Nakamura H, et al. Survey to determine the efficacy and safety of guideline-based pharmacological therapy for chronic obstructive pulmonary disease patients not previously receiving maintenance treatment. Expert Opin Pharmacother. 2015;16 (15):2271-2281. doi:10.1517/14656566.2015.1074678

21. Troosters T, Sciurba FC, Decramer M, et al. Tiotropium in patients with moderate COPD naive to maintenance therapy: a randomised placebo-controlled trial. NPJ Prim Care Respir Med. 2014;24:14003. doi:10.1038/npjpcrm.2014.3

22. Vogelmeier C, Fabbri LM, Rabe KF, et al. Effect of tiotropium vs. salmeterol on exacerbations: GOLD II and maintenance therapy naive patients. Respir Med. 2013;107(1):75-83. doi:10.1016/j.rmed. 2012.09.015
23. Decramer M, Rossi A, Lawrence D, McBryan D. Indacaterol therapy in patients with COPD not receiving other maintenance treatment. Respir Med. 2012;106(12):1706-1714. doi:10.1016/j.rmed.2012.08.022

24. Troosters T, Celli B, Lystig T, et al. Tiotropium as a first maintenance drug in COPD: secondary analysis of the UPLIFT trial. Eur Respir J. 2010;36(1):65-73. doi:10.1183/09031936.00127809

25. [Guideline for diagnosis and management of COPD (chronic obstructive pulmonary disease). The Third Edition: therapy and management]. Nihon Kokyuki Gakkai Zasshi. 2009;Suppl COPD:70-144. Available from: https://www.ncbi.nlm.nih.gov/pubmed/22712100. Accessed November $19,2019$.

26. Jones PW, Beeh KM, Chapman KR, Decramer M, Mahler DA, Wedzicha JA. Minimal clinically important differences in pharmacological trials. Am J Respir Crit Care Med. 2014;189(3):250-255. doi:10.1164/rccm.201310-1863PP

27. Price D, Miravitlles M, Pavord I, et al. First maintenance therapy for COPD in the UK between 2009 and 2012: a retrospective database analysis. NPJ Prim Care Respir Med. 2016;26:16061. doi:10.1038/npjpcrm.2016.61

28. Suissa S, Dell'Aniello S, Ernst P. Comparative effectiveness of LABA-ICS versus LAMA as initial treatment in COPD targeted by blood eosinophils: a population-based cohort study. Lancet Respir Med. 2018;6(11):855-862. doi:10.1016/S2213-2600(18)30368-0

29. Welte T, Vogelmeier C, Papi A. COPD: early diagnosis and treatment to slow disease progression. Int J Clin Pract. 2015;69(3):336-349. doi:10.1111/ijcp.2015.69.issue-3

30. Tantucci C, Modina D. Lung function decline in COPD. Int J Chron Obstruct Pulmon Dis. 2012;7:95-99. doi:10.2147/COPD

International Journal of Chronic Obstructive Pulmonary Disease

\section{Publish your work in this journal}

The International Journal of COPD is an international, peer-reviewed journal of therapeutics and pharmacology focusing on concise rapid reporting of clinical studies and reviews in COPD. Special focus is given to the pathophysiological processes underlying the disease, intervention programs, patient focused education, and self management protocols. This journal is indexed on PubMed Central, MedLine and CAS. The manuscript management system is completely online and includes a very quick and fair peer-review system, which is all easy to use. Visit http://www.dovepress.com/testimonials.php to read real quotes from published authors.

Submit your manuscript here: https:/www.dovepress.com/international-journal-of-chronic-obstructive-pulmonary-disease-journal 